\title{
Silenced SNHGI Inhibited Epithelial-Mesenchymal Transition and Inflammatory Response of ARPE-19 Cells Induced by High Glucose
}

\author{
Jing Yang (D) \\ Kun Yang ${ }^{2}$ \\ Xuxia Meng' \\ Penghui Liu' \\ Yudong Fu' \\ Yibo Wang' \\ 'Ophthalmology, Affiliated Hospital of \\ Qingdao University, Qingdao, 266500, \\ Shandong Province, People's Republic of \\ China; ${ }^{2}$ Central Laboratory, Affiliated \\ Hospital of Qingdao University, Qingdao, \\ 266500, Shandong Province, People's \\ Republic of China
}

Correspondence: Xuxia Meng Email mengxx@qduhospital.cn
Purpose: The lncRNA small nucleolar RNA host gene 1 (SNHG1) is a cerebral infarctionassociated gene, its biological role and mechanism in diabetic retinopathy remain to be illuminated. The present study was designed to investigate the role of SNHG1 in high glucose induced human retinal pigment epithelial cells (ARPE-19).

Methods: ARPE-19 cells were cultured and exposed to $60 \mathrm{mM}$ high glucose for $48 \mathrm{~h}$, and $5.5 \mathrm{mM}$ glucose-exposed ARPE-19 cells were used as the control. The levels of the epithelial-mesenchymal transition (EMT) markers E-cadherin, ZO-1, vimentin and $\alpha$-SMA were measured, and the Cell inflammatory response was evaluated by detecting IL- 6 and IL-1 $\beta$ levels. Then, cell migration, proliferation and apoptosis were detected. The expression of the lncRNA SNHG1 in ARPE-19 cells was detected by quantitative real-time PCR. SNHG1 was knocked down by small interfering RNA (siRNA) transfection. The effects of SNHG1 inhibition on inflammation, EMT, migration, proliferation and apoptosis were observed.

Results: The results showed that the expression of SNHG1 was significantly increased in ARPE-19 cells exposed to high glucose. Silencing SNHG1 reduced the expression of vimentin, $\alpha$-SMA, and the expression of inflammatory chemokines IL- 6 and IL-1 $\beta$, inhibited migration and proliferation, elevated the expression of E-cadherin and $\mathrm{ZO}-1$, and promoted apoptosis in ARPE-19 cells.

Conclusion: The lncRNA SNHG1 is involved in hyperglycemia-induced EMT and the inflammatory response of ARPE-19 cells and provides a new understanding of the pathogenesis of DR. Keywords: epithelial-mesenchymal transition, small nucleolar RNA host gene 1, retinal pigment epithelial, hyperglycemia

\section{Introduction}

Diabetic retinopathy (DR) is a common and specific microvascular complication of diabetes, causes vision-threatening retinal changes in one-third of the diabetic population, and remains the leading cause of blindness in working-age people. ${ }^{1-3}$ The pathogenesis of DR is relatively complex and has not been fully elucidated until now. There is evidence that the pathophysiology of DR is associated with hyperglycemia and its effect on retinal microvascular tissue. ${ }^{4}$ Proliferative diabetic retinopathy (PDR) is the main form of late-stage of $\mathrm{DR}$, and the main features are the changes in retinal microvessels, new blood vessels and the formation of fibrous proliferative anterior membrane. ${ }^{5}$

Retinal pigment epithelial (RPE) cells located between the sensory layer of the retinal nerve and the vascular choroid layer, are an important part of the blood- 
retina barrier and play an important role in maintaining the normal physiological function of the retina. Machemer proposed that RPE cell proliferation was the major factor in the formation of the proliferative membrane. ${ }^{6}$

Epithelial-mesenchymal transition (EMT) refers to the physiological or pathological process in which fully polarized cells anchored on the basement membrane under normal conditions undergo a variety of morphological and functional changes to acquire a mesenchymal phenotype, including the appearance of elongated spindle like morphology, enhanced migration and invasion ability, and enhanced antiapoptotic activity. ${ }^{7,8}$ EMT can be activated under pathological conditions, such as inflammation, wound healing, and carcinogenesis. ${ }^{9}$ A recent study has found that high glucose (HG) could induce EMT in RPE cells, which contributed to upregulation of the expression of fibrogenic factors. ${ }^{10,11}$ Therefore, it is speculated that EMT of RPE in PDR may be an important component of proliferative anterior retinal membrane formation.

Long nonprotein-coding RNAs (lncRNAs) have a wide range of cellular functions and are involved in almost all aspects of gene expression and protein translation and stability. $^{12}$

Exploring the function and potential mechanism of LncRNAs has been a hot research topic in recent years. The connection between lncRNAs and retinal impairments was explored further. ${ }^{13}$ The lncRNA small nucleolar RNA host gene 1 (SNHG1) is a new type of lncRNA located at chromosome $11 \mathrm{q} 12.3 .{ }^{14}$ It has been reported to be involved in the occurrence and development of several diseases, Further, according to previous studies, SNHG1 is aberrantly expressed in many cancers, including lung cancer, ${ }^{15}$ colorectal cancer, ${ }^{16}$ gastric cancer, ${ }^{17}$ and liver cancer, ${ }^{14}$ and promotes the EMT process in gastric cancer cells and colorectal cancer. ${ }^{18,19}$ However, its biological role and mechanism in DR remain to be elucidated.

Therefore, we attempted to further investigate the role of SNHG1 in ARPE-19 cells. In line with previous studies, we aimed to study the effect of SNHG1 on high glucoseinduced ARPE-19 cell injury. These findings might provide novel insight into DR treatment.

\section{Materials and Methods}

\section{ARPE-19 Cell Culture}

ARPE-19 cells are purchased from procell Life Technology Co., Ltd. (Wuhan, China). The complete culture medium for ARPE-19 cells consisted of Dulbecco's modified Eagle's medium (DMEM)/F-12 (volumetric ratio of 1:1; HyClone, Logan, UT, USA) and 10\% fetal bovine serum (FBS; HyClone). ARPE-19 cells were cultured at $37^{\circ} \mathrm{C}$ in humidified air with $5 \% \mathrm{CO} 2$. For treatment, ARPE-19 cells were grown in complete culture medium until approximately $75 \%$ confluence. Subsequently, the culture medium was replaced with FBS-free DMEM/ F-12 for $24 \mathrm{~h}$ before switching to high glucose $(60 \mathrm{mM}$ D-glucose) or normal glucose (NG) (5.5 mM D-glucose) conditions. The cell culture medium was not renewed during the $48 \mathrm{~h}$ high glucose and normal glucose treatment. When the cells were routinely cultured, the medium was renewed every other day.

\section{Cell Transfection}

High glucose-treated ARPE-19 cells were seeded onto 24well plates with antibiotic-free DMEM to $70 \%$ confluence before transfection. SNHG1 siRNA (si-SNHG1) and normal control siRNA (si-NC) were designed and synthesized by GenePharma (Shanghai, China). Cultured ARPE-19 cells were treated with the designed siRNAs. The siRNAs were transfected into ARPE-19 cells in serumfree Opti-MEM by using Lipofectamine 3000 (Invitrogen, Carlsbad, CA) for $48 \mathrm{~h}$.

\section{Quantitative Real-Time PCR Analysis}

Total RNA was extracted from cultured cells using TRIzol reagent according to the manufacturer's instructions (TaKaRa Bio Inc., Otsu, Japan). Total RNA (500 ng) was used for reverse transcription using the PrimeScript ${ }^{\circledR}$ RT reagent kit (Perfect Real-Time) (TaKaRa Bio Inc., Otsu, Japan). cDNA was used for quantitative PCR (qPCR) using SYBR ${ }^{\circledR}$ Premix Ex Taq ${ }^{\mathrm{TM}}$ (Perfect RealTime) (TaKaRa Bio Inc.) and a Roche capillary-based LightCycler $^{\circledR} \quad 2.0 \quad$ system (Roche Diagnostics, Indianapolis, IN, USA). The specificity of the amplification reactions was confirmed by melting curve analysis. The GAPDH gene was used as an internal reference, and the experiments were performed in triplicate ( 3 wells). The data were quantified by the comparative threshold cycle (Ct) method for relative gene expression. Three independent experiments were performed. The PCR cycling conditions were as follows: $95^{\circ} \mathrm{C}$ for $30 \mathrm{sec}, 95^{\circ} \mathrm{C}$ for $5 \mathrm{sec}$ and $60^{\circ} \mathrm{C}$ for $45 \mathrm{sec}$ for 40 cycles. Primer sequences were as follows: for GAPDH, forward

AACAGCCTCAAGATCATCAGCAA and reverse GACTGTGGTCATGAGTCCTTCCA; for 
SNHG1, forward GAGCCAATGAAACAGCAGT TGAG and reverse

TGGAAATCTGTGGTGAGAGGATCT.

\section{Cell Counting Kit-8 (CCK-8) Assay}

Cells treated with different concentrations of glucose (5.5 and $60 \mathrm{mmol} / \mathrm{L}$ ) were seeded in culture plates at $37^{\circ} \mathrm{C}$ for $48 \mathrm{~h}$. Then, the cells in each well of the plates were supplemented with CCK-8 solution (Dojindo Laboratories, Kumamoto, Japan) and cultured for at least $4 \mathrm{~h}$. A microplate reader (Bio-Rad) was utilized to estimate the absorbance at $450 \mathrm{~nm}$. In addition, a CCK-8 assay was also employed to determine the viability of ARPE-19 cells under different transfections after treatment with $60 \mathrm{mmol} / \mathrm{L}$ glucose for $48 \mathrm{~h}$. Three independent experiments were performed.

\section{Flow Cytometry}

Flow cytometry was used to evaluate the apoptosis of ARPE-19 cells. Briefly, the transfected cells were resuspended in $1 \times$ binding buffer and double-stained with the fluorescein isothiocyanate (FITC) Annexin V-propidium iodide (PI) kit (Meilunbio, LiaoNing, China). Finally, the FlowJo10.0.7 system was used to measure the apoptosis rate of APRE-19 cells according to the manufacturer's protocol. Three number of independent experiments was 3 .

\section{Wound Healing Assay}

Equal numbers of ARPE-19 cells and transfected ARPE19 cells were plated in each well of a 6-well plate until they reached approximately $80 \%$ confluence. The cells were starved in DMEM for $24 \mathrm{~h}$ and then exposed to L-glucose (as a control) and D-glucose $(60 \mathrm{mM})$ for 48 h. A horizontal line was drawn on the bottom of the well when the cells were fully confluent. The cells were washed 3 times with phosphate-buffered solution (PBS), placed under a microscope, and placed in serum-free medium in an incubator at $37^{\circ} \mathrm{C}$ and $5 \% \mathrm{CO} 2$. The distance traveled by the cells was measured every $12 \mathrm{~h}$. Three independent experiments were performed.

\section{ELISA Assay}

Levels of IL-6 (EL-R0015c, Elabscience) and IL-1 $\beta$ (ELR0012c, Elabscience) were detected by corresponding ELISA kit (Sigma-Aldrich, Darmstadt, Germany) according to the instructions. The optical density was examined at 450 $\mathrm{nm}$ with a Power Wave Microplate Reader (Bio-TEK, USA).The number of independent experiments was 3 .

\section{Western Blot Analysis}

After the indicated treatment, cells were lysed with RIPA buffer and centrifuged. The lysates were clarified by centrifugation at 12,000 rpm for $5 \mathrm{~min}$. The protein concentrations were measured by using a BCA kit (Pierce, Rockford, USA). Fifty micrograms of protein was loaded and separated on $6 \%$ and $10 \%$ SDS-PAGE gels and transferred onto nitrocellulose membranes (Bio-Rad, CA, USA). To avoid nonspecific binding, the membranes were blocked using $5 \%$ bovine serum albumin (BSA, Sigma-Aldrich, MO, USA) in PBS for 45 min at room temperature. The membranes were incubated overnight with primary antibodies, including anti-E-cadherin (AB-53267,1:1000, Elabscience), anti-vimentin (AB-70081, 1:1000, Elabscience), anti-zonula occludens-1 (ZO-1) (AB18170, 1:1000, Elabscience), anti- $\alpha$-SMA (AB-34268, 1:1000, Elabscience), anti-IL-6 (AB-19453,1:1000, Elabscience) and anti-IL- $\beta$ (AB-52153, 1:1000, Elabscience), with anti-GAPDH (AB-20072, 1:10,000; Elabscience) used as a loading control. After rinsing with PBS-T three times, the membranes were incubated with secondary antibodies (Elabscience) at room temperature for $2 \mathrm{~h}$. An antibody against GAPDH was used for the equivalent loading of total proteins. Protein bands were obtained using a FUSION FX7 Spectra (Vilber, France). Three independent experiments were performed.

\section{Statistical Analysis}

All of the data obtained from more than three independent experiments are expressed as the mean \pm standard deviation (SD). GraphPad Prism 8.0 (GraphPad Software, Inc., La Jolla, CA) was utilized to conduct statistical analyses. Comparisons between two groups were made by unpaired Student's $t$-test. $\mathrm{P}<0.05$ was considered statistically significant.

\section{Results \\ High Glucose Promoted EMT in ARPE-19 Cells}

We first evaluated the direct effect of high glucose in ARPE19 cells. After exposure to $60 \mathrm{mM}$ (high) glucose for $48 \mathrm{~h}$, ARPE-19 cells underwent mesenchymal transition. As shown in Figure 1, compared to exposure to L-glucose as a control, exposure to $60 \mathrm{mM}$ (high) glucose for $48 \mathrm{~h}$ elevated the levels of mesenchymal markers (VIM, $\alpha$-SMA) (Figure 1A-C) and decreased the levels of epithelial markers (E-cadherin, ZO-1-) in ARPE-19 cells (Figure 1A-C). 

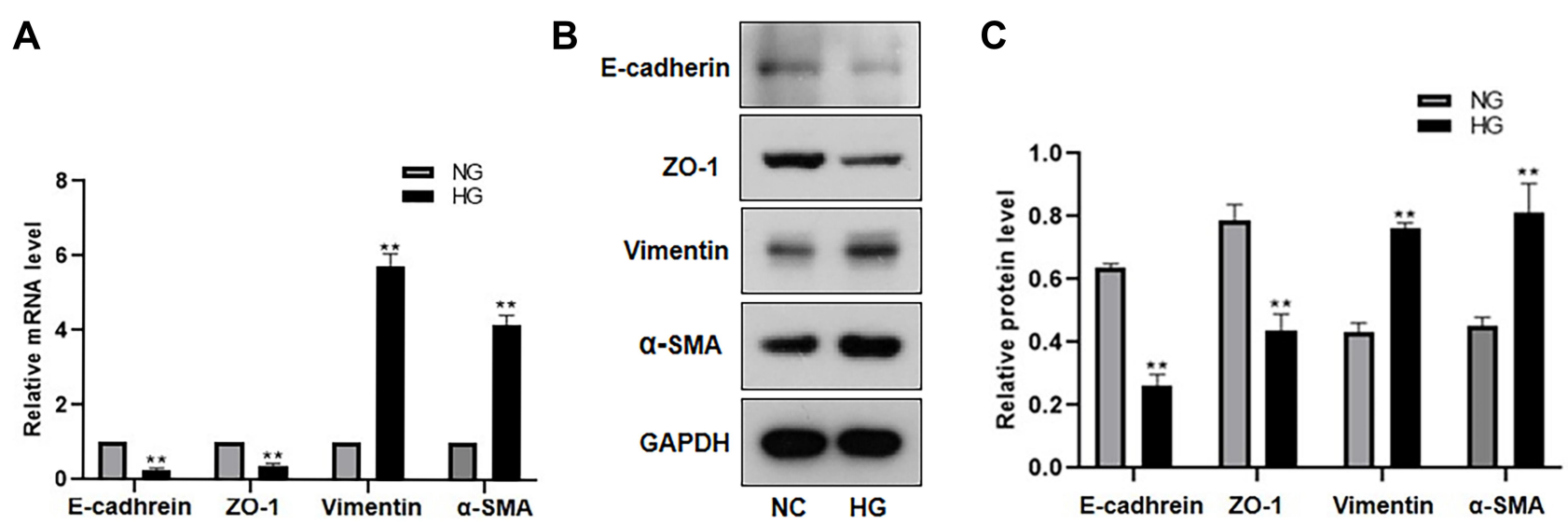

Figure I High glucose promoted EMT in ARPE-19 cells. ARPE-19 cells were cultured and exposed to $60 \mathrm{mM}$ high glucose for $48 \mathrm{~h}$, and $5.5 \mathrm{mM}$ glucose-exposed ARPE-19 cells were used as the control. (A) The mRNA level of EMT markers (E-cadherin, ZO-I, vimentin, $\alpha$-SMA) were detected by RT-PCR. **P < 0.0 I versus the control group. (B and C) The protein level of EMT markers (E-cadherin, ZO-I, vimentin, $\alpha$-SMA) were detected by Western blot. **P $<0.0$ I versus the control group. Data were shown as mean $\pm S D(n=3)$. Data obtained from more than three repeated experiments were shown as mean \pm SD.

\section{High Glucose Promoted the Migration and Proliferation of ARPE-19 Cells, Inhibited Apoptosis, and Increased the Expression of SNHGI}

Normal RPE cells are quiescent and lack proliferation and migration abilities. ${ }^{20}$ EMT can increase cell motility and proliferation. ${ }^{21}$ In our study, the mean number of migrated cells in the high glucose-exposed ARPE-19 cells was obviously higher than the mean number of migrated control cells (Figure 2A and B). The viability of high glucoseexposed ARPE-19 cells was significantly higher than that of migrated control cells (Figure 2C). Flow cytometry assays showed that high glucose inhibited the apoptosis of ARPE19 cells (Figure 2D and E). Furthermore, we detected SNHG1 expression in ARPE-19 cells exposed to $60 \mathrm{mM}$ (high) glucose for $48 \mathrm{~h}$ and found that SNHG1 expression was significantly increased (Figure 2F).

\section{Knocking-Down SNHGI Inhibited EMT in ARPE-19 Cells Induced by High Glucose}

We hypothesized that the upregulation of SNHG1 expression may be involved in the EMT of ARPE-19 cells. Therefore, we explored the role of SNHG1 in high glucoseinduced EMT in ARPE-19 cells by knocking out SNHG1. ARPE-19 cells were transfected with SNHG1-specific siRNA (si-SNHG1) or a negative control siRNA (Si-NC) after exposure to $60 \mathrm{mM}$ (high) glucose for $48 \mathrm{~h}$. SNHG1 expression was detected $48 \mathrm{~h}$ after transfection with siSNHG1, which decreased the expression of SNHG1 by more than $70 \%$ compared with si-NC (Figure 3A). We then detected the expression of EMT-related genes, including E-cadherin, zo-1, vimentin, and $\alpha$-SMA. Knockdown of SNHG1 significantly upregulated E-cadherin and ZO-1 expression and downregulated vimentin and $\alpha$-SMA expression (Figure 3B-D). These findings indicated that SNHG1 plays a critical role in high glucose-induced EMT.

\section{Knocking-Down SNHGI Inhibited the Migration and Proliferation of ARPE-19 Cells and Promoted the Apoptosis of ARPE-19 Cells}

We then explored the role of SNHG1 in the mobility and growth of ARPE-19 cells. The wound healing assay showed that downregulation of SNHG1 expression significantly inhibited the migration of RPE cells (Figure 4A and B). The CCK-8 assay showed that siRNA-mediated knockdown of SNHG1 significantly decreased the viability of ARPE-19 cells exposed to $60 \mathrm{mM}$ (high) glucose for 48 h (Figure 4C). Flow cytometry assays showed that knockdown of SNHG1 by siRNA promoted the apoptosis of ARPE-19 cells (Figure 4D and E).

\section{High Glucose Promoted the Expression of Proinflammatory Cytokines, Which Was Downregulated After SNHGI Knockdown}

Significant upregulation of the inflammatory cytokines IL-6 and IL-1 $\beta$ has been demonstrated in high glucose-induced RPE cells ${ }^{22,23}$ Studies have shown that SNHG1 attenuates 

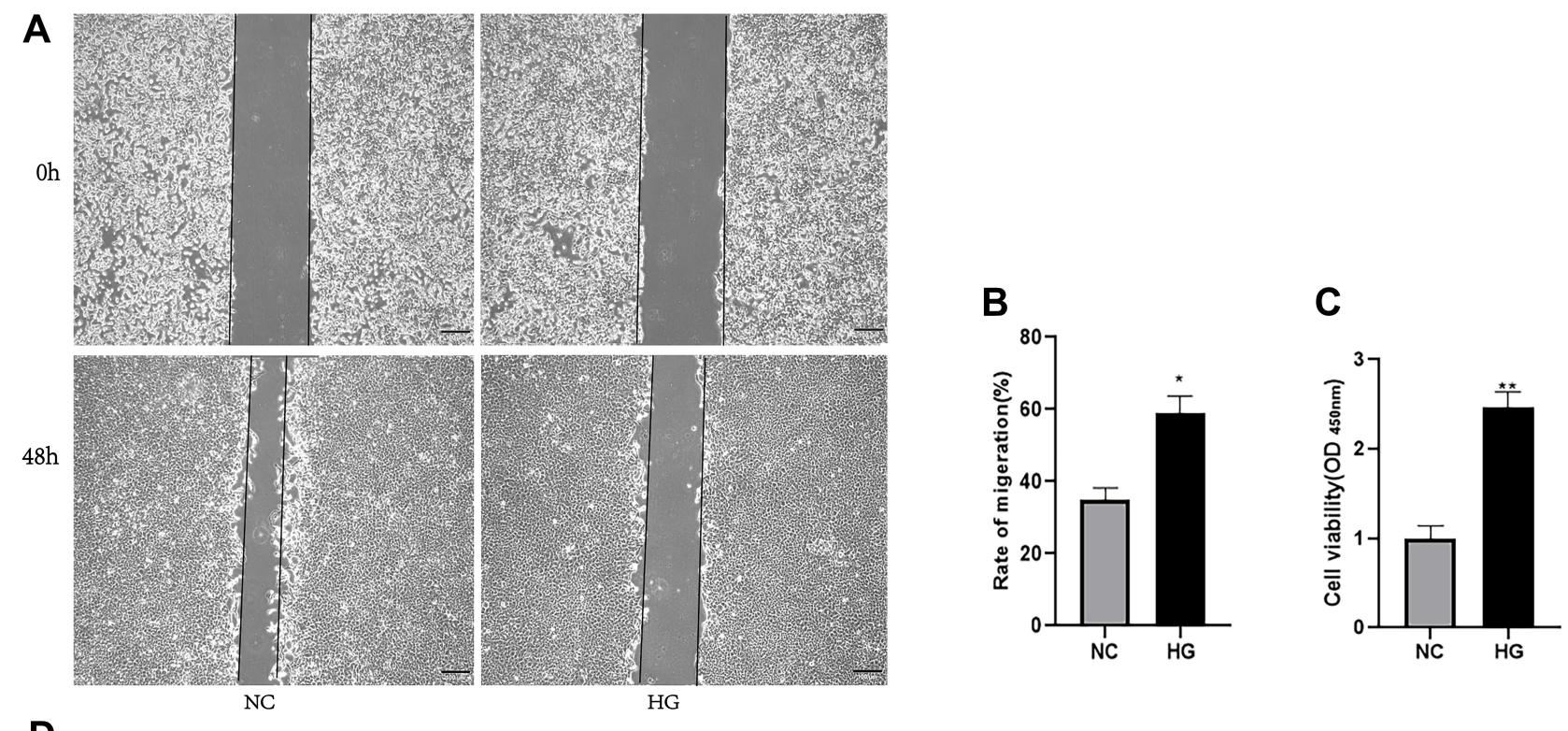

D

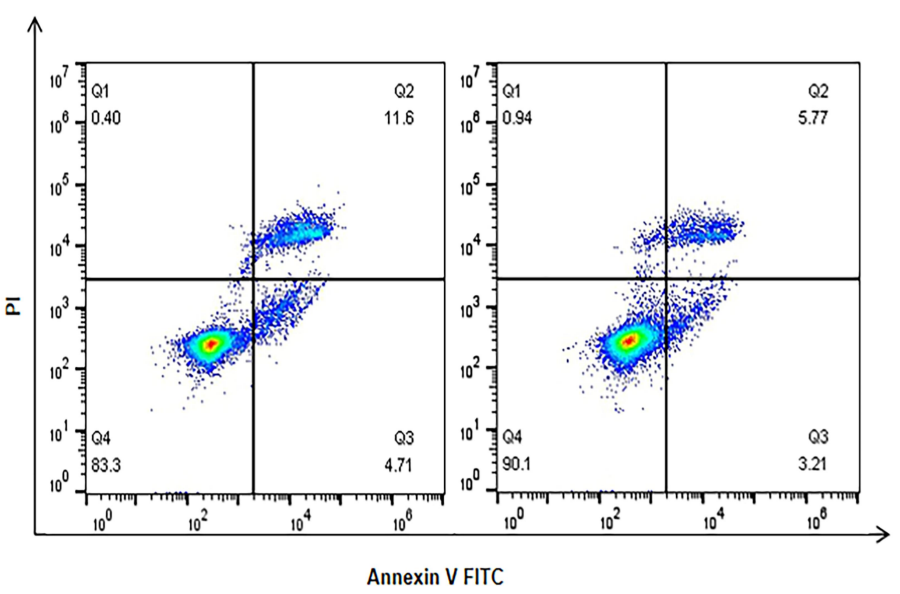

$\mathbf{E}$

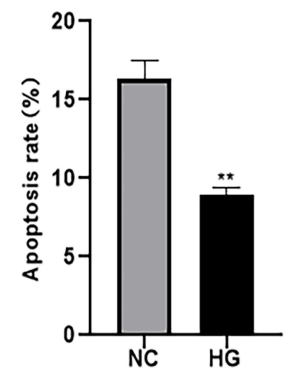

$\mathbf{F}$

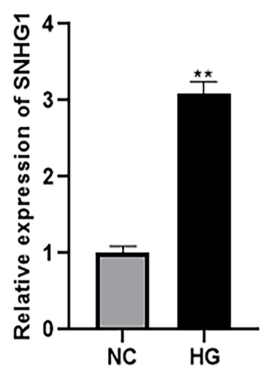

Figure 2 High glucose promoted the migration and proliferation of ARPE-19 cells, inhibited apoptosis, and increased the expression of SNHGI. ARPE-19 cells were cultured and exposed to $60 \mathrm{mM}$ high glucose for $48 \mathrm{~h}$, and $5.5 \mathrm{mM}$ glucose-exposed ARPE-19 cells were used as the control. (A and B) The migration rate was measured by the migration distance after a scratch was made. The data are presented as the means \pm SD, ${ }^{*} \mathrm{p}<0.05$ compared with L-glucose as the control. (C) CCK-8 assay were applied to determine cell viability. ${ }^{* *} \mathrm{p}<0.01$ compared with $\mathrm{L}$-glucose as the control. ( $\mathbf{D}$ and $\mathbf{E}$ ) Cell apoptosis rate was evaluated in flow cytometry analysis. $* * \mathrm{P}<0.0 \mathrm{I}$ compared with L-glucose as the control. (F) qRT-PCR assay revealed SNHGI expression. **P $<0.01$ compared with L-glucose as the control. Data obtained from more than three repeated experiments were shown as mean \pm SD.

cell injury and the inflammatory response in ox-LDL-induced human umbilical vein endothelial cells (HUVECs). ${ }^{24}$ To investigate the relationship between SNHG1 and inflammation in high glucose-induced ARPE-19 cells, inflammationrelated genes, including IL- 6 and IL- $1 \beta$, were analyzed by ELISA and Western blotting. IL-6 and IL-1 $\beta$ levels were significantly increased in ARPE-19 cells induced by high glucose, and knockdown of SNHG1 significantly downregulated the expression of IL-6 and IL-1 $\beta$ (Figure 5A-C).

\section{Discussion}

As an important part of the external retinal barrier, the RPE separates the neural retina from the fenestrated choroidal capillaries and plays an important role in regulating the flow of nutrients from the blood into photoreceptors and maintaining retinal adhesions. ${ }^{25}$ Under physiological conditions, the large amount of energy required for the metabolic activity of the sensory layer of retinal nerve is mainly provided by glucose that is transported by RPE cells from the choroidal capillaries, hence RPE cells are more susceptible to blood glucose fluctuations and can undergo a series of pathophysiological changes occur. ${ }^{26}$ Thiery et al reported that pathological events such as inflammation, senescence, and injury can initiate the migration and proliferation of RPE cells, which undergo EMT from polygonal-shaped epithelioid 
A

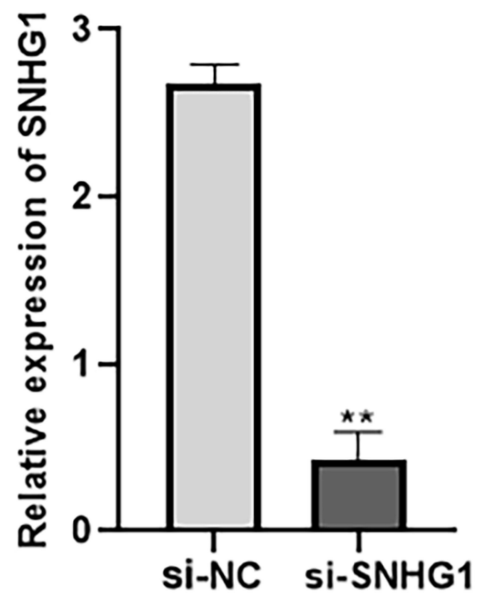

C

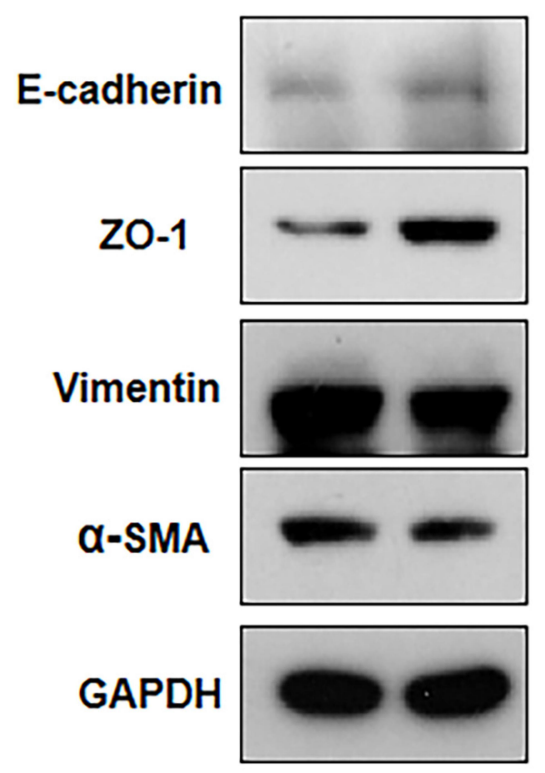

Si-NC Si-SNHG1
B

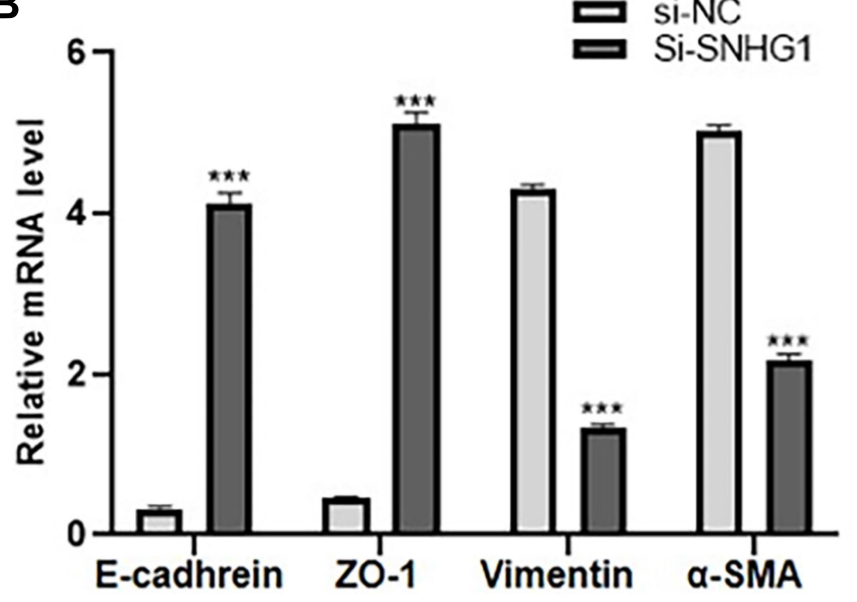

D

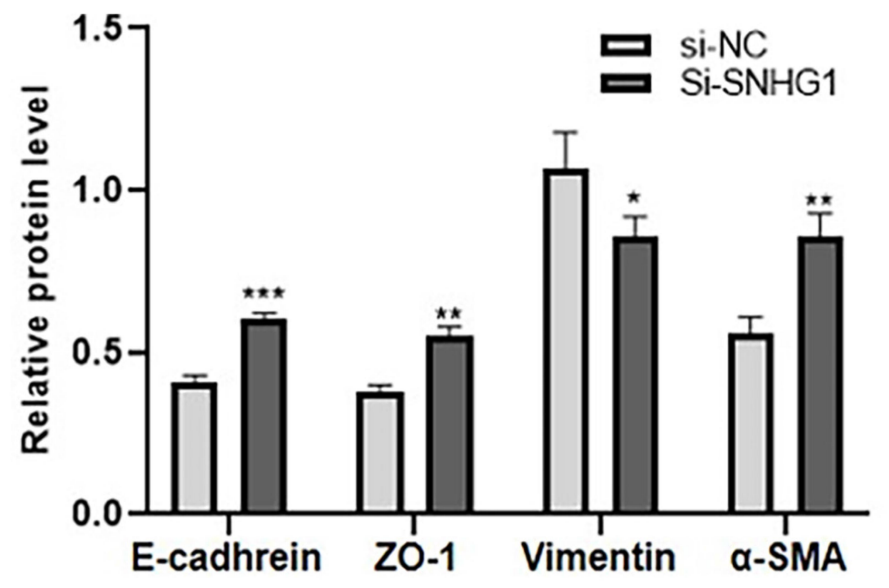

Figure 3 Knocking-down SNHGI inhibited EMT in ARPE- 19 cells induced by high glucose. ARPE-I 9 cells were transfected with SNHGI-specific siRNA (si-SNHGI) or a negative control siRNA (Si-NC) after exposure to $60 \mathrm{mM}$ (high) glucose for $48 \mathrm{~h}$. (A) qRT-PCR assay revealed SNHGI expression. **P < $0.0 \mathrm{l}$ compared with Si-NC as the control. (B) The mRNA level of EMT markers (E-cadherin, ZO-I, vimentin, $\alpha$-SMA) were detected by RT-PCR. ***P $<0.00 \mathrm{I}$ versus the control group. (C and D) The protein level of EMT markers (E-cadherin, ZO-I, vimentin, $\alpha$-SMA) were detected by Western blot. $* \mathrm{P}<0.05$, $* * \mathrm{P}<0.0 \mathrm{I}$ and $* * * \mathrm{P}<0.00 \mathrm{I}$ compared with Si-NC as the control. Data obtained from more than three repeated experiments were shown as mean \pm SD.

cells to fibroblasts or macrophages. ${ }^{8}$ Consistent with previous studies, ${ }^{10}$ the present study reported mesenchymal transformation in ARPE-19 cells exposed to high glucose. The Western blot and PCR results showed that the expression of epithelial markers E-Cad and ZO-1 decreased, and that of mesenchymal markers vimentin and $\alpha$-SMA increased. Among them, ZO- 1 is an indispensable tight junction protein for BRB integrity. ${ }^{27}$ Maintenance of BRB integrity is also due to the interaction between tight junctions and adhesion junctions mediated by cell-cell adhesion molecules, such as cadherin. ${ }^{28}$ Subsequently, ARPE-19 cells underwent migration and proliferation, and the apoptosis rate was significantly decreased. Our experiment proved that the tight junction of ARPE-19 cells changed at the level of mRNA and protein. Some experiments confirmed that when the tight junction of RPE cells changed, the transepithelial Electrical Resistance (TEER) of RPE cells would also change. ${ }^{29}$ In the later stage, we will continue to supplement the experiment to prove it. 

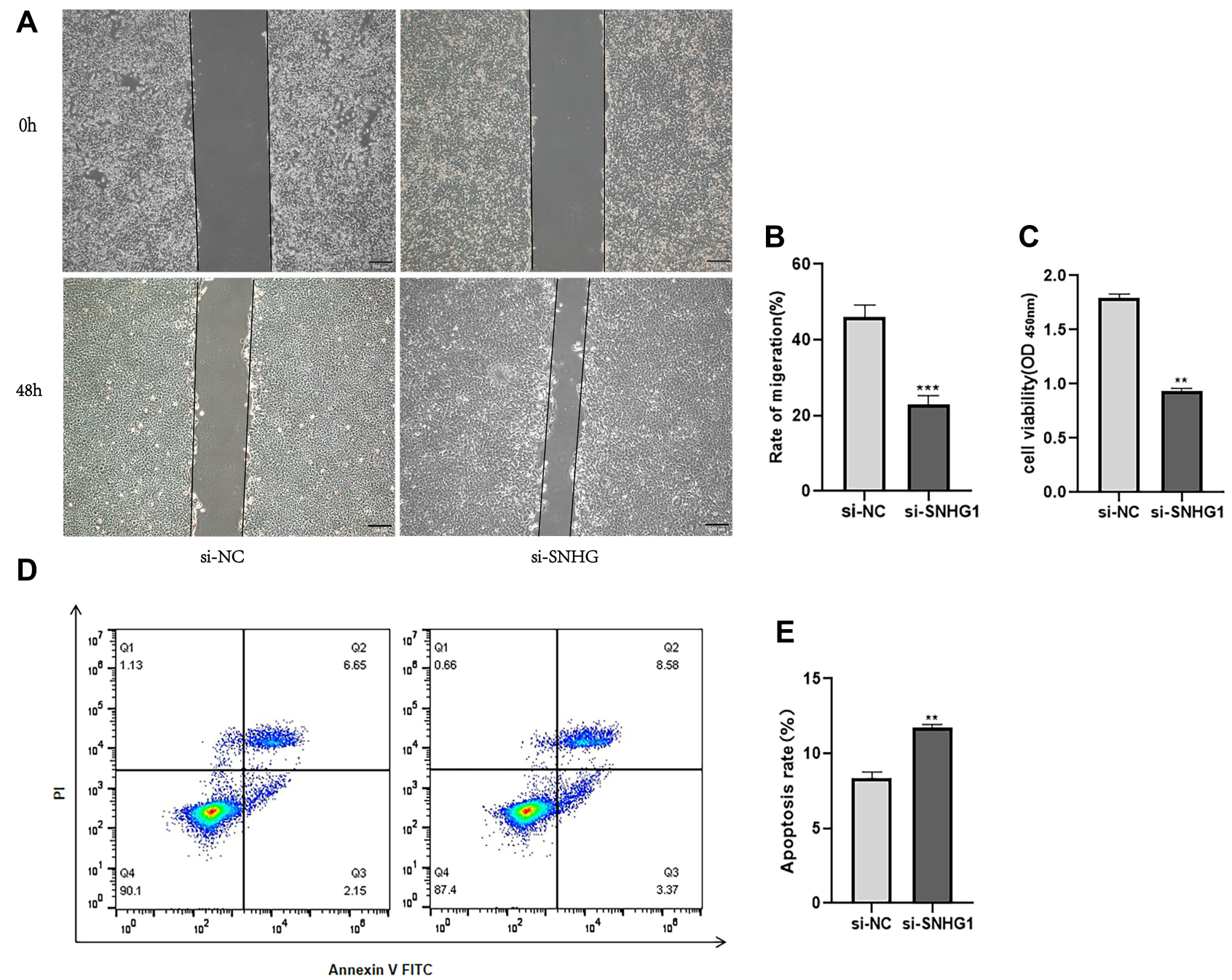

Figure 4 Knocking-down SNHGI inhibited the migration and proliferation of ARPE-19 cells and promoted the apoptosis of ARPE-19 cells. ARPE- 19 cells were transfected with SNHG I-specific siRNA (si-SNHGI) or a negative control siRNA (si-NC) after exposure to $60 \mathrm{mM}$ (high) glucose for $48 \mathrm{~h}$. (A and B) The migration rate was measured by the migration distance after a scratch was made. The data are presented as the means \pm SD, $* * * p<0.001$ compared with si-NC as the control. (C) CCK-8 assay were applied to determine cell viability. ${ }^{*}$ p $<0.01$ compared with si-NC as the control. (D and E) Cell apoptosis rate was evaluated in flow cytometry analysis. $* * \mathrm{P}<0.01$ compared with si-NC as the control.

The involvement of SNHG1 in the progression of multiple cancers has been confirmed. ${ }^{14}$ Zhao et al found that SNHG1 is highly expressed in renal carcinoma tissues and is associated with poor prognosis. Knockdown of SNHG1 suppressed the proliferation, invasion, and EMT capacity in renal carcinoma. ${ }^{30}$ Liu et al suggested that SNHG1 enhances the EMT process in gastric cancer cells through the DCLK1mediated Notch1 pathway. ${ }^{18}$ However, the relationship between SNHG1 and high glucose-induced RPE has not been reported. In this study, we demonstrated that hyperglycemia promoted SNHG1 expression. This result indicated that SNHG1 may play an essential role in the hyperglycemiainduced EMT of RPE cells. Therefore, we further explored the role of SNHG1 in ARPE-19 cells by knocking down SNHG1 using siRNA. We found that knockdown of SNHG1 significantly inhibited the high glucose-induced EMT phenotype, including the upregulation of E-cadherin and ZO-1, which are epithelial phenotypic markers, and the downregulation of vimentin and $\alpha$-SMA, which are mesenchymal phenotypic markers. Moreover, the migration and proliferation ability of ARPE-19 cells decreased, and the apoptosis rate increased. When EMT occurs, the migration, invasion and antiapoptotic ability of cells are enhanced. ${ }^{8,9}$ Our experimental results demonstrated that knockdown of SNHG1 can reverse EMT in ARPE-19 cells, thus reducing cell migration and proliferation and promoting apoptosis. Adherens and tightjunctions are crucial for BRB stability, it has been already proven that pharmacological intervention through inhibition of P2X7R restored expression of adherens and tight junctions in two models of inner blood retinal barrier. ${ }^{31}$ It has been 
A

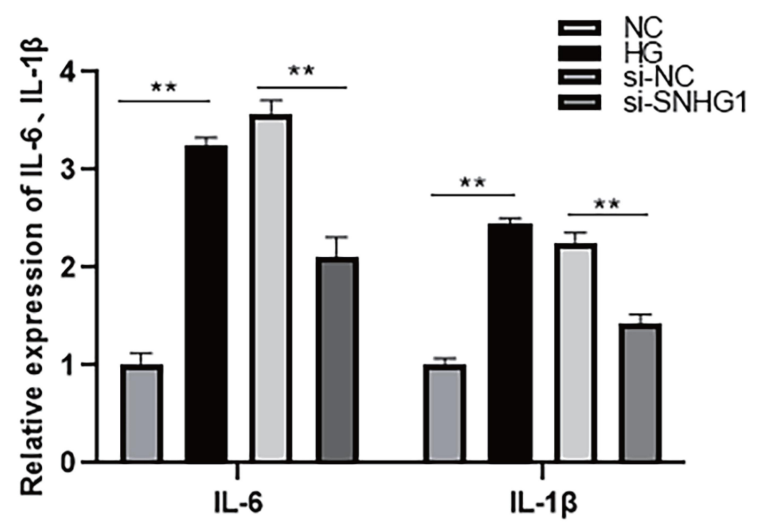

C

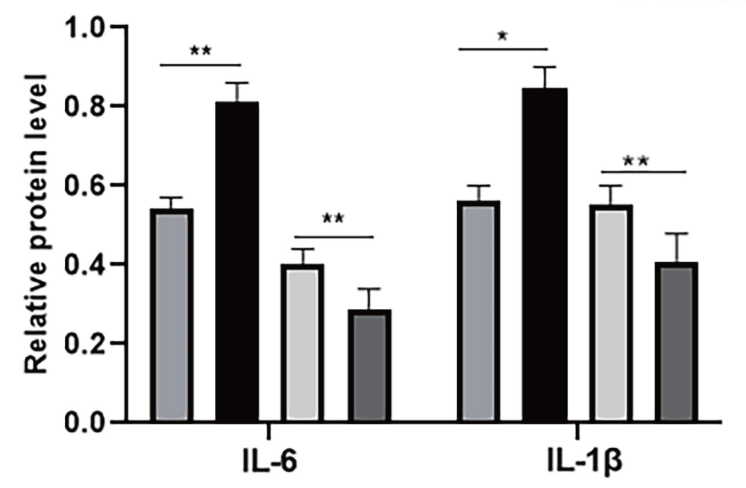

B

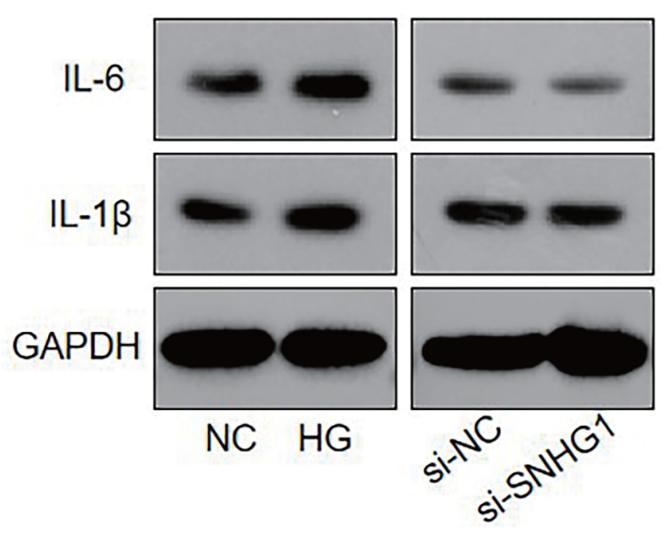

Figure 5 High glucose promoted the expression of proinflammatory cytokines, which was downregulated after SNHGI knockdown. (A) The level of IL-6 and IL-I $\beta$ were detected by ELASA. $* * P<0.01$ versus the control group. (B and $\mathbf{C}$ ) The protein level of IL-6 and IL-I $\beta$ were detected by Western blot. $* P<0.05$ and $* * P<0.0$ I versus the control group. Data obtained from more than three repeated experiments were shown as mean \pm SD.

confirmed that inhibiting the activation of Notch signal significantly weakens the EMT of RPE, ${ }^{32}$ so we speculate that SNHG1 promotes the EMT, of ARPE-19 cells by activating Notch signal pathway, but the specific mechanism remains to be further confirmed.

Increasing evidence indicates that inflammation plays an important role in the progression of DR. ${ }^{33}$ The levels of many inflammatory cytokines and chemokines, such as TNF- $\alpha$, IL- $1 \beta$ and IL-6, are elevated in serum and eye samples from diabetic patients with DR. ${ }^{34}$ The RPE is also a major source of proinflammatory mediators, and inflammatory RPE cell dysfunction may be related to DR. ${ }^{35,36}$ Previous studies have suggested that some proinflammatory stimuli may inhibit the barrier function of the RPE. ${ }^{37}$ Our experiments also confirmed that the expression of the inflammatory cytokines IL-6 and IL-1 $\beta$ was significantly increased in ARPE-19 cells induced by high glucose. IL-
$1 \beta$ is an important proinflammatory mediator secreted by activated macrophages during ocular inflammation. ${ }^{38}$ Under the induction of IL-1 $\beta$, the RPE is activated and produces proinflammatory cytokines such as IL-6, IL-8 and MCP-1. ${ }^{39}$ IL-6 is a multifunctional cytokine that plays a role in regulating immune processes and angiogenesis. PDR reports elevated levels of IL-6 in the eye fluid. ${ }^{13}$ Research by Lu et al showed that SNHG1 attenuated cell injury and the inflammatory response in ox-LDL-induced HUVECs by upregulating the expression of both GNAI2 and PCBP1 in a miR-556-5p-dependent manner. ${ }^{24}$ Therefore, we hypothesized that SNHG1 may also play a role in the increased levels of inflammatory cytokines in ARPE-19 cells exposed to high glycemia. We found that the expression of IL- 6 and IL- $1 \beta$ was significantly decreased in ARPE-19 cells induced by high glucose after SNHG1 knockdown. 
Most vision loss in DR patients occurs in the transition from inflammatory disease to neovascular fibrosis. ${ }^{40}$ Studies have confirmed that uncontrolled proliferation and migration of RPE cells can lead to the formation of pathological membranes on the surface of the neuroretina, resulting in retinal edema, detachment or degeneration and ultimately leading to visual impairment. ${ }^{41,42}$ Hiscott et al confirmed that RPE cells are part of the PDR proliferative membrane. ${ }^{43}$ In addition, RPE cells are involved in diabetic retinal neovascularization under the influence of traumatic and inflammatory conditions. ${ }^{44-46}$ We hypothesized that there was a correlation between the increase in inflammatory chemokine levels and the progression of EMT, and a number of experiments have confirmed this. Mesquida et al reported that the incubation of IL-6 on ARPE-19 cells for $48 \mathrm{~h}$ significantly destroyed the distribution of ZO-1 in ARPE- 19 cells. ${ }^{47}$ Jo et al found that IL6 increased VEGF mRNA expression in RPE cells to recruit microglia, and IL-6-treated microglia produced and secreted tumor necrosis factor, activated NF-kB, and reduced ZO-1 levels. ${ }^{48}$ Notch-1 and the nuclear factor Kappa light chain enhancer (NF- $\mathrm{BB})$, which activates $\mathrm{B}$ cells, have been reported to aggravate microgliamediated neuroinflammation. Activation of transmembrane Notch-1 receptor by inflammatory cytokines has been reported to be highly regulated by phosphorylation of NF- $\mathrm{kB} .{ }^{32}$ According to our experiment, we speculated that IL- 6 and IL-1 $\beta$ induced by high glucose activated Notch-1 signal pathway in ARPE-19 cells, thus promoting EMT in ARPE-19 cells, and further destroy the integrity of BRB. After SNHG1 silencing, the expression of IL-6 and IL-1 $\beta$ is decreased, which prevents the transformation from epithelium to stroma, but the exact mechanism needs to be further studied.

DR is mainly characterized by retinal microvascular changes and late proliferative membrane formation. The occurrence and development of EMT promotes the proliferation and migration of ARPE-19 cells, which induces the formation of proliferative membranes. We confirmed that SNHG1 was involved in the development of EMT and inflammation in high glucose-induced ARPE-19 cells. When SNHG1 is knocked down, EMT is reversed, inflammatory factors are downregulated, cell proliferation and migration are inhibited, and apoptosis is promoted.

In conclusion, our results confirmed that SNHG1 is a critical mediator in the EMT, inflammation, migration, proliferation and apoptosis of ARPE-19 cells. SNHG1 might play a significant role in the pathogenesis of DR.
The role of SNHG1 in activating RPE cells opens new windows for understanding the mechanisms of DR and may provide new potential therapeutic targets.

\section{Data Sharing Statement}

The raw data supporting the conclusions of this article will be made available by the authors, without undue reservation, to any qualified researcher. Readers can contact the corresponding author.

\section{Acknowledgments}

We sincerely appreciate all lab members.

\section{Author Contributions}

All authors made a significant contribution to the work reported, whether that is in the conception, study design, execution, acquisition of data, analysis and interpretation, or in all these areas; took part in drafting, revising or critically reviewing the article; gave final approval of the version to be published; have agreed on the journal to which the article has been submitted; and agree to be accountable for all aspects of the work.

\section{Funding}

Qingdao Science and Technology Project: Clinical study on the effect of Compound liquid of Traditional Chinese medicine on nosocomial infection control.

\section{Disclosure}

The authors report no conflicts of interest in this work.

\section{References}

1. Cheung N, Mitchell P, Wong TY. Diabetic retinopathy. Lancet. 2010;376(9735):124-136. doi:10.1016/S0140-6736(09)62124-3

2. Thomas AA, Biswas S, Feng B, Chen S, Gonder J, Chakrabarti S. IncRNA H19 prevents endothelial-mesenchymal transition in diabetic retinopathy. Diabetologia. 2019;62(3):517-530.

3. Liu Y, Song Y, Tao L, et al. Prevalence of diabetic retinopathy among 13473 patients with diabetes mellitus in China: a cross-sectional epidemiological survey in six provinces. BMJ Open. 2017;7(1):e013199. doi:10.1136/bmjopen-2016-013199

4. Abu-El-Asrar AM, Dralands L, Missotten L, Al-Jadaan IA, Geboes K. Expression of apoptosis markers in the retinas of human subjects with diabetes. Invest Ophthalmol Vis Sci. 2004;45(8):2760-2766. doi:10.1167/iovs.03-1392

5. Nawaz IM, Rezzola S, Cancarini A, et al. Human vitreous in proliferative diabetic retinopathy: characterization and translational implications. Prog Retin Eye Res. 2019;72:100756.

6. Machemer R, van Horn D, Aaberg TM. Pigment epithelial proliferation in human retinal detachment with massive periretinal proliferation. Am J Ophthalmol. 1978;85(2):181-191. doi:10.1016/ S0002-9394(14)75946-X 
7. Lamouille S, Xu J, Derynck R. Molecular mechanisms of epithelial-mesenchymal transition. Nat Rev Mol Cell Biol. 2014;15 (3):178-196.

8. Thiery JP, Acloque H, Huang RY, Nieto MA. Epithelialmesenchymal transitions in development and disease. Cell. 2009;139(5):871-890. doi:10.1016/j.cell.2009.11.007

9. Zhou M, Geathers JS, Grillo SL, et al. Role of epithelial-mesenchymal transition in retinal pigment epithelium dysfunction. Front Cell Dev Biol. 2020;8:501. doi:10.3389/fcell.2020.00501

10. Che D, Zhou T, Lan Y, et al. High glucose-induced epithelial-mesenchymal transition contributes to the upregulation of fibrogenic factors in retinal pigment epithelial cells. Int J Mol Med. 2016;38(6):1815-1822. doi:10.3892/ijmm.2016.2768

11. You ZP, Chen SS, Yang ZY, et al. GEP100/ARF6 regulates VEGFR2 signaling to facilitate high-glucose-induced epithelial-mesenchymal transition and cell permeability in retinal pigment epithelial cells. $\mathrm{Am}$ J Physiol Cell Physiol. 2019;316(6):C782-C791. doi:10.1152/ ajpcell.00312.2018

12. Schmitz SU, Grote P, Herrmann BG. Mechanisms of long noncoding RNA function in development and disease. Cell Mol Life Sci. 2016;73(13):2491-2509. doi:10.1007/s00018-016-2174-5

13. Donato L, Scimone C, Alibrandi S, et al. Transcriptome analyses of lncRNAs in A2E-stressed retinal epithelial cells unveil advanced links between metabolic impairments related to oxidative stress and retinitis pigmentosa. Antioxidants (Basel). 2020;9:undefined.

14. Thin KZ, Tu JC, Raveendran S. Long non-coding SNHG1 in cancer. Clin Chim Acta. 2019;494:38-47. doi:10.1016/j.cca.2019.03.002

15. Li Z, Lu Q, Zhu D, Han Y, Zhou X, Ren T. lnc-SNHG1 may promote the progression of non-small cell lung cancer by acting as a sponge of miR-497. Biochem Biophys Res Commun. 2018;506(3):632-640. doi:10.1016/j.bbrc.2018.10.086

16. Xu M, Chen X, Lin $\mathrm{K}$, et al. The long noncoding RNA SNHG1 regulates colorectal cancer cell growth through interactions with EZH2 and miR-154-5p. Mol Cancer. 2018;17(1):141. doi:10.1186/ s12943-018-0894-x

17. Guo W, Huang J, Lei P, Guo L, Li X. LncRNA SNHG1 promoted HGC-27 cell growth and migration via the miR-140/ADAM10 axis. Int $J$ Biol Macromol. 2019;122:817-823. doi:10.1016/j.ijbiomac.2018.10.214

18. Liu ZQ, He WF, Wu YJ, et al. LncRNA SNHG1 promotes EMT process in gastric cancer cells through regulation of the miR-15b/ DCLK1/Notch1 axis. BMC Gastroenterol. 2020;20(1):156. doi:10.1186/s12876-020-01272-5

19. Bai J, Xu J, Zhao J, Zhang R. IncRNA SNHG1 cooperated with miR-497/miR-195-5p to modify epithelial-mesenchymal transition underlying colorectal cancer exacerbation. J Cell Physiol. 2020;235 (2):1453-1468. doi:10.1002/jcp.29065

20. Bharti K, Nguyen MT, Skuntz S, Bertuzzi S, Arnheiter H. The other pigment cell: specification and development of the pigmented epithelium of the vertebrate eye. Pigment Cell Res. 2006;19(5):380-394. doi:10.1111/j.1600-0749.2006.00318.x

21. Hazan RB, Phillips GR, Qiao RF, Norton L, Aaronson SA. Exogenous expression of $\mathrm{N}$-cadherin in breast cancer cells induces cell migration, invasion, and metastasis [published correction appears in J Cell Biol 2000 Apr 3; 149(1): following236]. J Cell Biol. 2000;148(4):779-790. doi:10.1083/jcb.148.4.779

22. Luo R, Li L, Hu YX, Xiao F. LncRNA H19 inhibits high glucose-induced inflammatory responses of human retinal epithelial cells by targeting miR-19b to increase SIRT1 expression [published online ahead of print, 2020 Oct 6]. Kaohsiung J Med Sci. 2020;37 (2):101-110. doi:10.1002/kjm2.12302

23. Cunha-Vaz J, Bernardes R, Lobo C. Blood-retinal barrier. Eur J Ophthalmol. 2011;21(Suppl 6):S3-S9. doi:10.5301/EJO.2010.6049

24. Lu Y, Xi J, Zhang Y, et al. SNHG1 inhibits ox-LDL-induced inflammatory response and apoptosis of HUVECs via up-regulating GNAI2 and PCBP1. Front Pharmacol. 2020;11:703. doi:10.3389/fphar.2020.00703
25. Lakkaraju A, Umapathy A, Tan LX, et al. The cell biology of the retinal pigment epithelium [published online ahead of print, $2020 \mathrm{Feb}$ 24]. Prog Retin Eye Res. 2020;100846.

26. Yao Y, Guan M, Zhao XQ, Huang YF. Hypoxia and high glucose concentration on human retinitis pigmentosa in vitro culture. Chin J Med. 2003;83:1989-1992.

27. Runkle EA, Antonetti DA. The blood-retinal barrier: structure and functional significance. Methods Mol Biol. 2011;686:133-148.

28. Campbell HK, Maiers JL, DeMali KA. Interplay between tight junctions \& adherens junctions. Exp Cell Res. 2017;358(1):39-44. doi:10.1016/j.yexcr.2017.03.061

29. Zou XL, Wang GF, Li DD, et al. Protection of tight junction between RPE cells with tissue factor targeting peptide. Int J Ophthalmol. 2018;11(10):1594-1599. doi:10.18240/ijo.2018.10.04

30. Zhao S, Wang Y, Luo M, Cui W, Zhou X, Miao L. Long noncoding RNA small nucleolar RNA host gene 1 (SNHG1) promotes renal cell carcinoma progression and metastasis by negatively regulating miR-137. Med Sci Monit. 2018;24:3824-3831. doi:10.12659/MSM.910866

31. Fresta CG, Caruso G, Fidilio A, et al. Dihydrotanshinone, a natural diterpenoid, preserves blood-retinal barrier integrity via P2X7 receptor. Int J Mol Sci. 2020;21(23):9305. doi:10.3390/ ijms21239305

32. Anchi P, Swamy V, Godugu C. Nimbolide exerts protective effects in complete Freund's adjuvant induced inflammatory arthritis via abrogation of STAT-3/NF- $\mathrm{BB} /$ Notch-1 signaling. Life Sci. 2021;266:118911. doi:10.1016/j.1fs.2020.118911

33. Adamis AP. Is diabetic retinopathy an inflammatory disease? $\mathrm{Br}$ J Ophthalmol. 2002;86(4):363-365. doi:10.1136/bjo.86.4.363

34. Rübsam A, Parikh S, Fort PE. Role of inflammation in diabetic retinopathy. Int $J$ Mol Sci. 2018;19(4):942. doi:10.3390/ ijms 19040942

35. Forrester JV, Kuffova L, Delibegovic M. The role of inflammation in diabetic retinopathy. Front Immunol. 2020;11:583687. doi:10.3389/ fimmu.2020.583687

36. Samuels IS, Bell BA, Pereira A, Saxon J, Peachey NS. Early retinal pigment epithelium dysfunction is concomitant with hyperglycemia in mouse models of type 1 and type 2 diabetes. $J$ Neurophysiol. 2015;113(4):1085-1099. doi:10.1152/jn.00761.2014

37. Shirasawa M, Sonoda S, Terasaki H, et al. TNF- $\alpha$ disrupts morphologic and functional barrier properties of polarized retinal pigment epithelium. Exp Eye Res. 2013;110:59-69. doi:10.1016/j. exer.2013.02.012

38. Kuppner MC, McKillop-Smith S, Forrester JV. TGF-beta and IL-1 beta act in synergy to enhance IL-6 and IL-8 mRNA levels and IL-6 production by human retinal pigment epithelial cells. Immunology. 1995;84(2):265-271.

39. Elner VM, Scales W, Elner SG, Danforth J, Kunkel SL, Strieter RM. Interleukin-6 (IL-6) gene expression and secretion by cytokine-stimulated human retinal pigment epithelial cells. Exp Eye Res. 1992;54(3):361-368. doi:10.1016/0014-4835(92)90048-W

40. Kim SJ, Kim YS, Kim JH, et al. Activation of ERK1/2-mTORC1NOX4 mediates TGF- $\beta 1$-induced epithelial-mesenchymal transition and fibrosis in retinal pigment epithelial cells. Biochem Biophys Res Commun. 2020;529(3):747-752. doi:10.1016/j.bbrc.2020.06.034

41. Pastor JC, de la Rúa ER, Martín F. Proliferative vitreoretinopathy: risk factors and pathobiology. Prog Retin Eye Res. 2002;21 (1):127-144. doi:10.1016/S1350-9462(01)00023-4

42. Simó R, Villarroel M, Corraliza L, Hernández C, Garcia-Ramírez M. The retinal pigment epithelium: something more than a constituent of the blood-retinal barrier-implications for the pathogenesis of diabetic retinopathy. J Biomed Biotechnol. 2010;2010:190724. doi:10.1155/ 2010/190724

43. Hiscott P, Gray R, Grierson I, Gregor Z. Cytokeratin-containing cells in proliferative diabetic retinopathy membranes. $\mathrm{Br} J$ Ophthalmol. 1994;78(3):219-222. doi:10.1136/bjo.78.3.219 
44. Saika S, Yamanaka O, Okada Y, et al. TGF beta in fibroproliferative diseases in the eye. Front Biosci (Schol Ed). 2009;1(1):376-390. doi:10.2741/s32

45. Scimone C, Alibrandi S, Scalinci SZ, et al. Expression of pro-angiogenic markers is enhanced by blue light in human RPE cells. Antioxidants (Basel). 2020;9(11):1154. doi:10.3390/antiox9111154

46. Donato L, Scimone C, Alibrandi S, et al. Possible A2E mutagenic effects on RPE mitochondrial DNA from innovative RNA-seq bioinformatics pipeline. Antioxidants (Basel). 2020;9(11):1158. doi:10.3390/antiox9111158
47. Mesquida M, Drawnel F, Lait PJ, et al. Modelling macular edema: the effect of IL-6 and IL-6R blockade on human blood-retinal barrier integrity in vitro. Transl Vis Sci Technol. 2019;8(5):32. doi:10.1167/tvst.8.5.32

48. Jo DH, Yun JH, Cho CS, Kim JH, Kim JH, Cho CH. Interaction between microglia and retinal pigment epithelial cells determines the integrity of outer blood-retinal barrier in diabetic retinopathy. Glia. 2019;67(2):321-331. doi:10.1002/glia.23542

\section{Publish your work in this journal}

The Journal of Inflammation Research is an international, peerreviewed open-access journal that welcomes laboratory and clinical findings on the molecular basis, cell biology and pharmacology of inflammation including original research, reviews, symposium reports, hypothesis formation and commentaries on: acute/chronic inflammation; mediators of inflammation; cellular processes; molecular mechanisms; pharmacology and novel anti-inflammatory drugs; clinical conditions involving inflammation. The manuscript management system is completely online and includes a very quick and fair peerreview system. Visit http://www.dovepress.com/testimonials.php to read real quotes from published authors. 